\title{
ALTERAÇÕES DO SOLO INFLUENCIADAS PELO TRÁFEGO E CARGA DE UM "FORWARDER" NAS ENTRELINHAS DE UMA FLORESTA DE EUCALIPTO ${ }^{(1)}$
}

\author{
Sérgio Ricardo Silva ${ }^{(2)}$, Nairam Félix de $\operatorname{Barros}^{(3)}$, Liovando Marciano \\ da Costa ${ }^{(3)}$, Eduardo de Sá Mendonça $^{(3)}$ \& Fernando Palha Leite ${ }^{(4)}$
}

\begin{abstract}
RESUMO
Durante a retirada de madeira de povoamentos florestais as máquinas trafegam numa mesma linha várias vezes, o que pode causar a compactação do solo. O grau de compactação, além do número de passadas da máquina, pode também ser afetado pelo peso de madeira transportado. Este trabalho teve como objetivo avaliar a compactação do solo de acordo com a intensidade de tráfego e a carga de madeira de um "forwarder". O estudo foi realizado no município de Santana do Paraíso, MG, em um Latossolo Amarelo caulinítico. Um povoamento de eucalipto de seis anos de idade com espaçamento de $3 \times 2 \mathrm{~m}$ foi cortado por motosserra, sendo a madeira retirada da área manualmente. Em seguida, demarcaram-se parcelas experimentais compostas por quatro entrelinhas de árvores com $12 \mathrm{~m}$ de comprimento, com área de $144 \mathrm{~m}^{2}$ cada. Dois experimentos foram realizados. No primeiro, testou-se o efeito da intensidade de tráfego, no qual um "forwarder" (modelo Valmet $636 \mathrm{~S}$ ), com tara de 11,9 t, foi carregado com $8 \mathrm{~m}^{3}$ de madeira (densidade de $480 \mathrm{~kg} \mathrm{~m}^{-3}$ ) e trafegou sobre a mesma entrelinha por 0,4 , 8 e 12 vezes. No segundo, $o$ "forwarder" recebeu cargas correspondentes a $0,4,8$ e $12 \mathrm{~m}^{3}$ de madeira e trafegou quatro vezes sobre a mesma entrelinha. Em cada parcela, quatro entrelinhas foram compactadas pelas rodas. $\mathrm{O}$ teor de $\mathrm{C}$ orgânico era de 2,68 e 2,40 dag $\mathrm{kg}^{-1}$, e a umidade atual do solo, de 15,6 e 17,1 dag kg-1, nas camadas de 0-10 e 10-20 cm de profundidade, respectivamente, correspondendo a 78 e $86 \%$ do equivalente de umidade. $O$ teor de argila era de $510 \mathrm{~g} \mathrm{~kg}^{-1}$ e o de areia, $450 \mathrm{~g} \mathrm{~kg}^{-1}$. As avaliações realizadas após a aplicação dos tratamentos, nas camadas de 0-10 e 10-20 cm de profundidade, foram: densidade do solo e agregados estáveis em água. Determinou-se, ainda, a resistência à penetração até à profundidade de $60 \mathrm{~cm}$, com o uso de um penetrômetro de impacto, e a percentagem da área superficial coberta com resíduos florestais, pelo método da corda. Verificou-se
\end{abstract}

\footnotetext{
${ }^{(1)}$ Parte da Tese de Doutorado do primeiro autor submetida à Universidade Federal de Viçosa (MG). Trabalho apresentado no XXIX Congresso Brasileiro de Ciência do Solo, em Ribeirão Preto (SP), em 13-18 de julho de 2003. Financiado pela CAPES. Recebido para publicação em abril de 2004 e aprovado em janeiro de 2007.

(2) Engenheiro-Agrônomo, Veracel Celulose S.A. Caixa Postal 21, CEP 45820-970 Eunápolis (BA). E-mail: sergio.silva@veracel.com.br

(3) Professor do Departamento de Solos da Universidade Federal de Viçosa. Av P.H. Rolfs s/n, CEP 36570-000 Viçosa (MG). E-mail: nfbarros@ufv.br; liovando@solos.ufv.br; esm@ufv.br

(4) Engenheiro-Agrônomo, Celulose Nipo Brasileira. Rod. BR 381, Km 172, CEP 35196-000 Belo Horiente (MG). E-mail: fernando.leite@cenibra.com.br
} 
que o tráfego do "forwarder" aumentou a densidade e a resistência do solo à penetração, reduziu a estabilidade de agregados em água e a cobertura do solo. A maior parte dos efeitos da compactação foi manifestada por apenas quatro passadas do "forwarder", mesmo quando vazio.

Termos de indexação: densidade do solo, agregados estáveis em água, resistência do solo à penetração, colheita florestal.

\title{
SUMMARY: SOIL DISTURBANCES AS AFFECTED BY LOAD AND TRAFFIC INTENSITY OF A FORWARDER ALONG INTERROWS OF A EUCALYPT STAND
}

\begin{abstract}
For the timber exploitation of forest stands, transport machines pass several times along the same track and this operation can cause soil compaction. This paper aimed to evaluate the soil compaction level as related to the number of passes of a forwarder and weight of the timber load. The study was carried out in Santana do Paraíso country, Minas Gerais State, Brazil, in a six year-old eucalypt stand with $3 \times 2 \mathrm{~m}$ spacing on an caulinitic Oxisol. The trees were felled by a chainsaw and removed from the area by hand. Then plots of $144 \mathrm{~m}^{2}$ (four tree interrows $12 \mathrm{~m}$ long) were outlined to conduct two trials;. In the first, the number of passes of a forwarder loaded with weights of 11.9 tons and $8 \mathrm{~m}^{3}$ (wood density $480 \mathrm{~kg} \mathrm{~m}^{-3}$ ) was tested in the following situations: no passes (wood removed by hand), 4, 8, and 12 passes. In the second trial, forwarder wood loads of $0,4,8$, and $12 \mathrm{~m}^{3}$ were tested by driving the machine four times along the same track. In each plot, the passes affected four tree interrows. The soil of the area is clayey (510 $\left.\mathrm{g} \mathrm{kg}^{-1} \mathrm{clay}\right)$ and the moisture content during the trial was 15.6 and $17.1 \mathrm{dag} \mathrm{kg}^{-1}$, at depths of $0-10 \mathrm{~cm}$ and 10 $20 \mathrm{~cm}$, respectively. These layers were assessed for soil density and water-stable aggregates. Soil penetration resistance was evaluated up to a depth of $60 \mathrm{~cm}$. Soil surface coverage by the harvest residues was also evaluated. Forwarder traffic increased the soil compaction and penetration resistance and reduced aggregate stability and soil coverage. Even with an empty forwarder, strong disturbances were observed after only four passes.
\end{abstract}

Index terms: Soil bulk density, water-stable aggregates, soil penetration resistance, forest harvest.

\section{INTRODUÇÃO}

O constante e significativo aumento do peso dos veículos agrícolas e florestais, registrado nas últimas décadas, preocupa pelas conseqüências em longo prazo sobre a produtividade dos solos transitados, com implicações na conservação dos recursos naturais. Em razão disso, o potencial de prejuízos à produtividade florestal é grande, já que essa operação se repete de forma recorrente (Balbuena et al., 2000).

$\mathrm{O}$ tráfego repetido em uma mesma área provoca danos à estrutura do solo, com conseqüente redução no rendimento tanto no primeiro ano de produção como nos anos posteriores (Håkansson \& Reeder, 1994; Lal, 1996; Jorajuria et al., 1997). Durante a retirada de madeira de povoamentos florestais, as máquinas trafegam numa mesma linha várias vezes, o que pode causar a compactação do solo e, como conseqüência, facilitar o processo erosivo e dificultar o crescimento de raízes. O grau de compactação, além do número de passadas, pode também ser influenciado pelo peso de madeira transportada; a deformação ocorrerá quando as pressões aplicadas ao solo excederem sua capacidade de suporte de carga (Dias Júnior, 2000).
A compactação causa mudanças desfavoráveis na densidade, porosidade, permeabilidade e resistência do solo à penetração (Alakukku \& Elonen, 1994; Ishaq et al., 2001a). A resistência à penetração aumenta com a compactação do solo, sendo restritiva ao crescimento radicular acima de valores, que variam de 1,5 a 3,0 MPa, conforme Grant \& Lafond (1993), e de 2,0 a 4,0 MPa, segundo Oussible et al. (1992) e Arshad et al. (1996).

Jakobsen \& Greacen (1985), estudando a compactação causada por "forwarder" em solos com plantio de pínus, observaram que os valores de resistência do solo à penetração eram acrescidos de 0,2 a $0,3 \mathrm{MPa}$ a cada passada da máquina e que a densidade do solo aumentava e a condutividade hidráulica diminuía com o número de passadas.

A importância da estabilidade de agregados na manutenção de propriedades físicas do solo diante de fatores adversos, como a colheita e o cultivo mecanizados, tem despertado o interesse de vários pesquisadores (Unger, 1995; Levy \& Miller, 1997), uma vez que ela se correlaciona bem com a permeabilidade e a resistência do solo à erosão hídrica. 
Este trabalho teve como objetivo avaliar os efeitos da compactação ocasionada pela intensidade de tráfego e carga de madeira de um "forwarder" sobre a cobertura e as propriedades do solo.

\section{MATERIAL E MÉTODOS}

O estudo foi realizado no município de Santana do Paraíso, MG (19 ${ }^{\circ} 22^{\prime} 58^{\circ}$ " S e $42^{\circ} 28^{\prime} 56^{\prime}$ W), em um Latossolo Amarelo caulinítico em relevo ondulado com declividade de $17^{\circ}$. O teor de $\mathrm{C}$ orgânico (Yeomans \& Bremner, 1988) era de 2,68 e 2,40 dag kg-1 e a umidade do solo (determinada por gravimetria), de 15,6 e 17,1 dag kg-1, nas camadas de 0-10 e 10-20 cm de profundidade, respectivamente, correspondendo a 78 e $86 \%$ do equivalente em umidade (água retida no solo sob tensão de $0,033 \mathrm{MPa}$ ). O teor de argila era de $510 \mathrm{~g} \mathrm{~kg}^{-1}$, e o de areia, $450 \mathrm{~g} \mathrm{~kg}^{-1}$ (método do densímetro - Embrapa, 1997).

Um povoamento de eucalipto com seis anos de idade plantado no sentido da declividade do terreno (espaçamento entre as linhas de $3 \mathrm{~m}$ e entre as plantas na linha de $2 \mathrm{~m}$ ) foi cortado por motosserra, sendo a madeira retirada da área manualmente. Em seguida, demarcaram-se quatro blocos em faixas transversais à declividade do terreno (em curva de nível). Cada bloco possuía 32 linhas de árvores e seis árvores por linha, totalizando 192 árvores e uma área de $1.152 \mathrm{~m}^{2}$; portanto, a área experimental total foi de $4.608 \mathrm{~m}^{2}$. Dois experimentos foram desenvolvidos em delineamento em blocos casualizados, com quatro repetições. No primeiro, testou-se o efeito da intensidade de tráfego, no qual um trator florestal autocarregável ("forwarder", marca Valmet, modelo $636 \mathrm{~S}$, largura total de $2,74 \mathrm{~m}$, rodas com $0,6 \mathrm{~m}$ de largura, velocidade de trabalho em $1^{\mathrm{a}}$ marcha de $7,4 \mathrm{~km} \mathrm{~h}^{-1}$, potência de $83 \mathrm{~kW}$ a $2.300 \mathrm{rpm}$ ), com tara de $11,9 \mathrm{t}$, foi carregado com $8 \mathrm{~m}^{3}$ de madeira (densidade de $480 \mathrm{~kg} \mathrm{~m}^{-3}$ ) e transitado sobre a mesma entrelinha por 0 (tratamento sem tráfego), 4, 8 e 12 vezes. A área compactada em cada entrelinha possuía $1 \mathrm{~m}$ de largura, ou seja, a soma da largura das duas trilhas formadas pela passagem das rodas da máquina. No segundo, o "forwarder" recebeu cargas correspondentes a 0 (vazio), 4, 8 e $12 \mathrm{~m}^{3}$ de madeira e trafegou quatro vezes sobre a mesma entrelinha. Portanto, foram aplicados oito tratamentos na área experimental. Em cada tratamento com tráfego do "forwarder", quatro entrelinhas foram compactadas pelas rodas da máquina, totalizando oito trilhas de tráfego por tratamento, das quais apenas as quatro centrais foram utilizadas para avaliações e as demais bordaduras. O comprimento de cada trilha no bloco foi de $12 \mathrm{~m}$, dos quais apenas $8 \mathrm{~m}$ centrais foram usados para avaliação. Assim, a área útil de cada parcela compunha-se das duas entrelinhas de plantio centrais $(6 \mathrm{~m})$ com quatro árvores $(8 \mathrm{~m})$, totalizando $48 \mathrm{~m}^{2}$. As amostragens foram realizadas nas trilhas de passagem dos rodados do "forwarder".
As avaliações realizadas nas camadas de 0-10 e 10-20 cm de profundidade, no centro da trilha formada pelas rodas do "forwarder", foram: densidade do solo e agregados estáveis em água (Embrapa, 1997), sendo obtido o diâmetro médio geométrico segundo Kemper \& Rosenau (1986). Determinou-se, ainda, a resistência à penetração até a profundidade de $60 \mathrm{~cm}$, com o uso de um penetrômetro de impacto (modelo IAA/PLANALSUCAR - STOLF (Stolf et al., 1983), e a percentagem da área superficial coberta com resíduos florestais, após uma chuva de $56 \mathrm{~mm}$ ocorrida no dia seguinte à instalação do experimento, pelo método da corda (Olszevski et al., 1998). Este método consistiu no uso de uma corda com comprimento de $10 \mathrm{~m}$, marcada a cada $25 \mathrm{~cm}$ (ou 1 ponto) com uma cor que contrastou com a da corda e com a da área a ser avaliada. A corda foi estendida sobre as trilhas, em três posições aleatórias em cada parcela, e a avaliação da cobertura foi feita considerando a área coberta por resíduos vegetais ou por solo exposto.

Os dados de densidade (individualmente, por camada: 0-10 e 10-20 cm de profundidade) e cobertura do solo foram submetidos às análises de variância e de regressão. Ajustaram-se os modelos de regressão linear, quadrático e quadrático base raiz quadrada. Para escolha dos modelos, os coeficientes foram testados pelo teste F ( $p<0,01$ e p $<0,05)$, com base no quadrado médio do resíduo da análise de variância. Finalmente, para dois ou mais modelos com coeficientes significativos, a seleção foi feita com base no maior $\mathrm{R}^{2}$. Os dados de agregados estáveis em água foram submetidos à análise de variância, e as médias, comparadas pelo teste de agrupamento Scott-Knott $(\mathrm{p}<0,05)$.

\section{RESULTADOS E DISCUSSÃO}

Houve aumento de até 15,3\% na densidade do solo, em relação ao tratamento sem tráfego, devido à compactação ocasionada pelas rodas do "forwarder" nas profundidades de 0-10 (oito passadas) e de 10-20 cm (quatro passadas) (Figura 1). No entanto, as diferenças entre os valores de densidade obtidos com 4,8 e 12 passadas foram pequenas, atingindo 2,5 e 4,9\%, respectivamente, nas camadas de 0-10 e 10-20 cm de profundidade. Jorajuria \& Draghi (2000) relataram que $90 \%$ do incremento máximo medido para a densidade aparente na camada superficial (0-30 cm) foi produzido já na primeira passada de um trator leve. De acordo com outros autores, a primeira passada é considerada aquela que induz as maiores mudanças na estrutura do solo, o que se restringe a condições de solos recém-lavrados (Taylor et al., 1982). No entanto, quando o solo se encontra consolidado, o grau de compactação pode ser similar para as distintas passadas. Koger et al. (1985) constataram que a maior parte da compactação total ocasionada pelo tráfego de um "skidder" ocorria nas primeiras passadas da 
máquina. Seixas \& Souza (1998) observaram que cerca de $80 \%$ da compactação total do solo, ocorrida após 20 passadas de um trator mais carreta carregada de madeira, resultou das cinco primeiras viagens desse equipamento, não havendo incremento devido ao tráfego subseqüente.

Não foi verificado efeito do aumento da carga ( 0,4 , 8 e $12 \mathrm{~m}^{3}$ de madeira) sobre a densidade do solo, que teve média de 1,22 e 1,24 $\mathrm{kg} \mathrm{dm}^{-3}$, respectivamente, nas camadas de $0-10$ e $10-20 \mathrm{~cm}$ de profundidade. No entanto, a densidade do solo nesses tratamentos foi 9,9 e 11,7 \% maior em relação ao tratamento sem tráfego nas camadas de $0-10$ e $10-20 \mathrm{~cm}$, respectivamente. Para Greacen \& Sands (1980), o acréscimo da carga não aumenta, necessariamente, a compactação, pois pode ocorrer aumento da superfície de contato pneu/solo, resultando na redistribuição da carga. Apesar de essa mensuração da superfície de contato pneu/solo não ter sido realizada no presente trabalho, trata-se de uma provável explicação para os resultados apresentados.

Houve redução dos agregados pertencentes à classe de 4,75-2 mm de diâmetro em decorrência do tráfego do "forwarder" (Quadro 1). Essa redução foi, em média, de 11,5 e $25,2 \%$ nas profundidades de $0-10$ e 10 $20 \mathrm{~cm}$, respectivamente, isto é, aproximadamente 2,2 vezes menor na camada superficial do solo. Isso proporcionou, na maioria das vezes, o aumento dos agregados nas demais classes, especialmente na profundidade de 10-20 $\mathrm{cm}$. Em virtude dessa redistribuição dos agregados entre classes de tamanho, observa-se que maiores incrementos ocorreram nas classes $2-1$ e $<0,5 \mathrm{~mm}$, respectivamente nas profundidades de $0-10$ e 10-20 cm, indicando, portanto, menor estabilidade de agregados do solo da camada subsuperficial. Não houve diferença significativa entre os valores de agregados, nas respectivas classes, obtidos nos tratamentos com diferentes números de passadas $(4,8$ e 12) e também entre os tratamentos com cargas variadas $\left(0,4,8\right.$ e $12 \mathrm{~m}^{3}$ de madeira), o que significa que a maior parte dos efeitos da compactação foi manifestada por apenas quatro passadas do "forwarder", mesmo quando vazio. De acordo com Koger et al. (1985) e Nugent et al. (2003), as passadas iniciais, durante um tráfego de máquinas, são as responsáveis pelo maior incremento da compactação do solo, em relação às passadas seguintes. Nota-se, ainda, a maior percentagem de agregados maiores (4,75-2 mm de diâmetro) na profundidade de 0-10 cm, o que pode ser atribuído ao maior teor de C orgânico $\left(2,68\right.$ dag $\left.\mathrm{kg}^{-1}\right)$ nesta camada em relação à de 10-20 cm (2,40 dag $\left.\mathrm{kg}^{-1}\right)$, proporcionando maior efeito agregante do solo na camada superficial.

Em decorrência da redistribuição dos agregados entre classes de tamanho, em resposta à compactação do solo, ocorreu decréscimo significativo de até 19,7\%

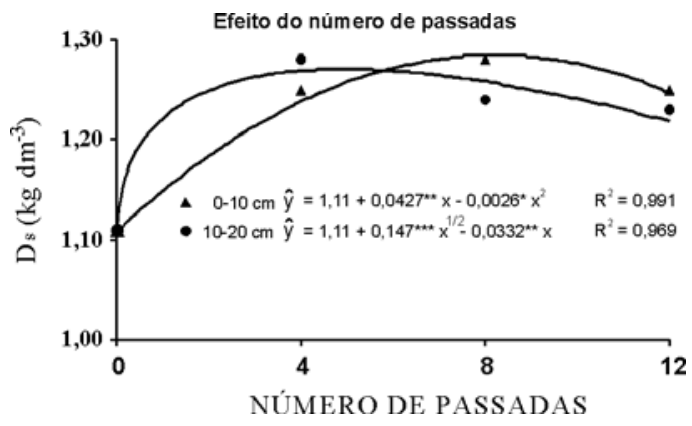

Figura 1. Densidade do solo resultante do número de passadas do "forwarder" em povoamento de eucalipto, num Latossolo Amarelo caulinítico, em Santana do Paraíso, MG.

Quadro 1. Distribuição do tamanho e diâmetro médio geométrico (DMG) de agregados estáveis em água afetados pelo número de passadas e pela carga do "forwarder" em povoamento de eucalipto, em Latossolo Amarelo caulinítico, em Santana do Paraíso-MG

\begin{tabular}{|c|c|c|c|c|c|c|c|c|c|c|}
\hline \multirow{2}{*}{$\begin{array}{c}\text { Tratamento } \\
\text { (passadas) }\end{array}$} & \multicolumn{4}{|c|}{ Profundidade: $0-10 \mathrm{~cm}$} & \multirow{2}{*}{ DMG } & \multicolumn{4}{|c|}{ Profundidade: $10-20 \mathrm{~cm}$} & \multirow{2}{*}{ DMG } \\
\hline & $4,75-2 \mathrm{~mm}$ & $2-1 \mathrm{~mm}$ & $1-0,5 \mathrm{~mm}$ & $<0,5 \mathrm{~mm}$ & & $4,75-2 \mathrm{~mm}$ & $2-1 \mathrm{~mm}$ & $1-0,5 \mathrm{~mm}$ & $<0,5 \mathrm{~mm}$ & \\
\hline & $\%$ & & & $\%$ & $\mathrm{~mm}$ & $\%$ & & & $\%$ & $\mathrm{~mm}$ \\
\hline \multicolumn{11}{|c|}{ Efeito do número de passadas } \\
\hline 0 & $91,8 \mathrm{a}$ & $5,5 \mathrm{~b}$ & $1,0 \mathrm{a}$ & $1,7 \mathrm{a}$ & $1,61 \mathrm{a}$ & 88,2 a & $6,5 \mathrm{~b}$ & $2,0 \mathrm{~b}$ & $3,3 \mathrm{~b}$ & $1,57 \mathrm{a}$ \\
\hline 4 & $77,4 \mathrm{~b}$ & $10,6 \mathrm{a}$ & $4,1 \mathrm{a}$ & $7,9 \mathrm{a}$ & $1,43 \mathrm{a}$ & $68,7 \mathrm{~b}$ & $11,1 \mathrm{a}$ & $6,8 \mathrm{a}$ & $13,4 \mathrm{a}$ & $1,32 \mathrm{~b}$ \\
\hline 8 & $80,4 \mathrm{~b}$ & $10,7 \mathrm{a}$ & $3,9 \mathrm{a}$ & $5,0 \mathrm{a}$ & $1,49 \mathrm{a}$ & $64,1 \mathrm{~b}$ & $14,6 \mathrm{a}$ & $7,2 \mathrm{a}$ & $14,1 \mathrm{a}$ & $1,29 \mathrm{~b}$ \\
\hline 12 & $82,8 \mathrm{~b}$ & 10,5 a & $2,4 \mathrm{a}$ & $4,3 \mathrm{a}$ & $1,52 \mathrm{a}$ & $62,7 \mathrm{~b}$ & $14,6 \mathrm{a}$ & 9,6 a & $13,1 \mathrm{a}$ & $1,28 \mathrm{~b}$ \\
\hline \multicolumn{11}{|c|}{ Efeito da carga } \\
\hline Média $^{(1)}$ & 82,0 & 9,4 & 3,2 & 5,4 & 1,49 & 66,5 & 12,7 & 7,7 & 13,1 & 1,31 \\
\hline
\end{tabular}

Valores seguidos pela mesma letra na coluna, individualmente para cada experimento e para cada profundidade, não diferem entre si a $5 \%$.

(1) É apresentada para o experimento de carga somente a média dos tratamentos com $0,4,8$ e $12 \mathrm{~m}^{3}$ de madeira, pois não houve efeito significativo destes tratamentos sobre as classes de agregados nem sobre o DMG. 
do diâmetro médio geométrico (DMG) de agregados estáveis em água da camada de $10-20 \mathrm{~cm}$ de profundidade (Quadro 1). No entanto, na camada de $0-10 \mathrm{~cm}$ não foi constatada diferença $(r<0,05)$ entre os tratamentos. Esse fato pode ser atribuído ao maior teor de matéria orgânica na camada superficial do solo, pois, segundo Tisdall \& Oades (1982), ela atua no aumento da estabilidade de agregados, devido ao seu efeito de agregação dos constituintes minerais do solo. Campos et al. (1995) encontraram correlação positiva $(r=0,85)$ entre DMG dos agregados e teor de matéria orgânica. Os agregados estáveis em água indicam, indiretamente, a qualidade estrutural do solo (Silva et al., 2000) e sua susceptibilidade à erosão hídrica. Por isso, a matéria orgânica aumenta a resistência do solo aos efeitos deletérios da compactação em sistemas mecanizados de colheita florestal.

A resistência do solo à penetração $(\mathrm{RP})$ aumentou com o número de passadas (Figura 2), como também verificado em outros trabalhos (Marsili \& Servadio, 1996; Marsili et al., 1998). É interessante observar que esse efeito se localizou especialmente na camada de $5-25 \mathrm{~cm}$ de profundidade, a exemplo do que foi obtido por Nugent et al. (2003), os quais verificaram maior aumento da RP pelo tráfego de um "forwarder" na profundidade de $10-30 \mathrm{~cm}$, atribuindo esse efeito ao fato de esta camada de solo estar mais consolidada estruturalmente. De acordo com outros pesquisadores, o peso da máquina é o fator principal que determina a profundidade onde a compactação do solo será maior, independentemente da pressão aplicada na sua superfície (Jorajuria et al., 1997; Jorajuria \& Draghi, 2000). A passagem do implemento na mesma entrelinha por 4, 8 e 12 vezes ocasionou aumentos da $\mathrm{RP}$, a $10 \mathrm{~cm}$ de profundidade, de 5,6; 4,7 e 7,5 vezes, respectivamente, em relação à testemunha nãocompactada (0 passada). Resultados semelhantes foram obtidos por Balbuena et al. (2000), que relataram serem os aumentos na RP mais importantes na camada superficial (nos primeiros $40 \mathrm{~cm}$ de profundidade), sendo incrementados com o acréscimo do número de passagens do trator. Não ocorreu grande variação da RP entre os valores obtidos nas quatro cargas utilizadas (Figura 2). Portanto, o efeito do número de passadas é maior do que o efeito da carga.

Os valores máximos de RP foram de 8,65 e 6,46 $\mathrm{MPa}$, respectivamente nos experimentos de intensidade de tráfego e carga, sendo consideravelmente maiores que os 2,87 MPa obtidos por Fernandes \& Souza (2003) nas trilhas, depois da última passada do "forwarder". É importante destacar que qualquer RP superior a $2 \mathrm{MPa}$ pode reduzir o crescimento e desenvolvimento radicular (Oussible et al., 1992; Martino \& Shaykewich, 1994; Ishaq et al., 2001b).

Neste trabalho, a profundidade de $5-25 \mathrm{~cm}$ foi a melhor para avaliação da compactação do solo por meio do uso de penetrômetro. Segundo Raper \& Erbach (1990), para um dado solo, a distribuição da compactação no perfil se dá de acordo com a carga aplicada, a pressão exercida pelo rodado, a umidade do solo e a intensidade de tráfego recebida.

O tráfego do "forwarder" ocasionou a redução da cobertura do solo nas trilhas dos pneus, cuja avaliação foi realizada após uma chuva de $56 \mathrm{~mm}$ ocorrida no dia seguinte à instalação do experimento (Figura 3). Essa redução foi devido ao efeito dilacerante dos pneus sobre a serapilheira e à redução da infiltração de água no solo, resultando em aumento do escoamento superficial e conseqüente erosão hídrica, favorecidos pela declividade do terreno $\left(17^{\circ}\right)$, como observado in situ. O aumento do número de passadas proporcionou redução de até 51,7 \% (12 passadas) da cobertura do solo em relação ao tratamento sem tráfego. Não houve diferença significativa entre os valores de cobertura do solo obtidos nos tratamentos de carga $(0,4,8$ e $\left.12 \mathrm{~m}^{3}\right)$. No entanto, o valor médio de $72,3 \%$ de cobertura do solo nestes tratamentos foi $24,8 \%$ menor em relação ao do tratamento sem tráfego.

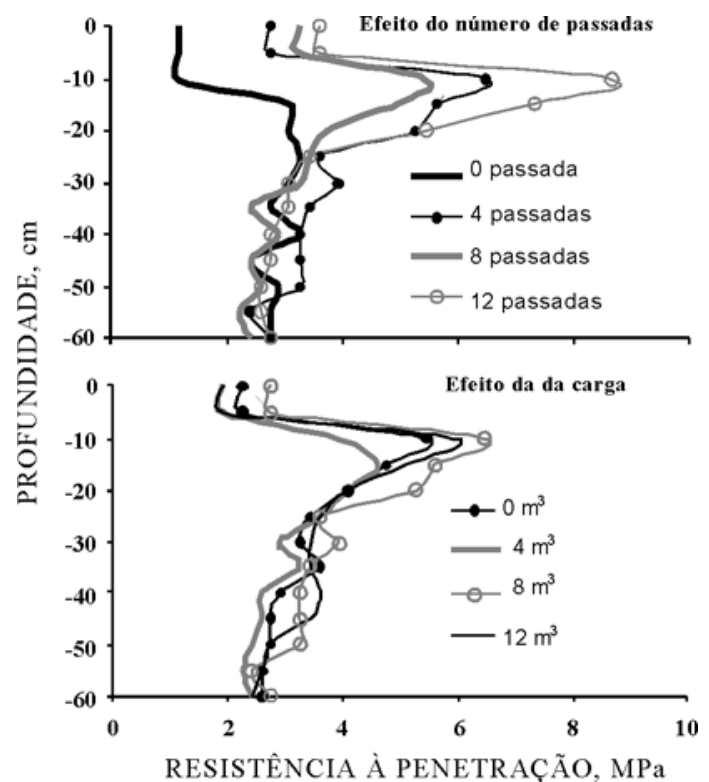

Figura 2. Resistência do solo à penetração de acordo com o número de passadas e a carga do "forwarder" em povoamento de eucalipto, num Latossolo Amarelo caulinítico, em Santana do Paraíso, MG.

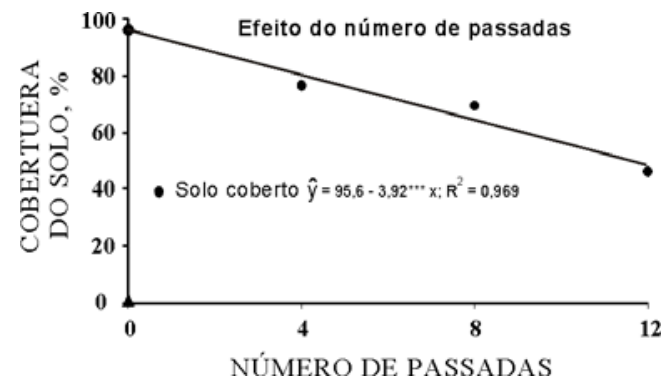

Figura 3. Cobertura do solo relacionada ao número de passadas do "forwarder" em povoamento de eucalipto, num Latossolo Amarelo caulinítico, em Santana do Paraíso, MG. 


\section{CONCLUSÕES}

1. O tráfego do "forwarder" aumentou a densidade e a resistência do solo à penetração, bem como reduziu a estabilidade de agregados em água e a cobertura do solo.

2. A maior parte dos efeitos da compactação foi manifestada por apenas quatro passadas do "forwarder", mesmo quando vazio.

\section{LITERATURA CITADA}

ALAKUKKU, L. \& ELONEN, P. Finish experiment on subsoil compaction by vehicles with high axle load. Soil Till. Res., 29:151-155, 1994.

ARSHAD, M.A.; LOWERY, B. \& GROSSMAN, B. Physical tests for monitoring soil quality. In: DORAN, J.W. \& JONES, A.J., eds. Methods for assessing soil quality. Madison, Soil Science Society of America, 1996. p.123141. (SSSA Special Publication, 49).

BALBUENA, R.H.; TERMINIELLO, A.M.; CLAVERIE, J.A.; CASADO, J.P. \& MARLATS, R. Compactación del suelo durante la cosecha forestal. Evolución de las propriedades físicas. R. Bras. Eng. Agric. Amb., 4:453-459, 2000.

CAMPOS, B.C.; REINERT, D.J.; NICOLODI, R.; RUEDELL, J. \& PETRERE, C. Estabilidade estrutural de um Latossolo Vermelho-Escuro distrófico após sete anos de rotação de culturas e sistemas de manejo de solo. R. Bras. Ci. Solo, 19:121-126, 1995.

DIAS JÚNIOR, M.S. Compactação do solo. In: NOVAIS, R.F.; ALVAREZ V., V.H. \& SCHAEFER, C.E.G.R., eds. Tópicos em ciência do solo. Viçosa, MG, Sociedade Brasileira de Ciência do Solo, 2000. v.1. p.55-94.

EMPRESA BRASILEIRA DE PESQUISA AGROPECUÁRIA EMBRAPA. Centro Nacional de Pesquisa de Solos. Manual de métodos de análise de solo. 2.ed. Rio de Janeiro, 1997. 212 p.

FERNANDES, H.C. \& SOUZA, A.P. Compactação de um Latossolo Vermelho causada pelo tráfego do "Forwarder". R. Árvore, 27:279-284, 2003.

GRANT, C.A. \& LAFOND, G.P. The effects of tillage systems and crop sequences on soil bulk density and penetration resistence on a clay soil in Southern Saskatchewan. Can. J. Soil Sci., 73:223-232, 1993.

GREACEN, E.L. \& SANDS, R. Compaction of forest soils: A review. Aust. J. Soil Res., 18:163-89, 1980.

HÅKANSSON, I. \& REEDER, R.C. Subsoil compaction by vehicles with high axle load-extent, persistence and crop response. Soil Till. Res., 29:277-304, 1994.

ISHAQ, M.; HASSAN, A.; SAEED, M.; IBRAHIM, M. \& LAL, R. Subsoil compaction effects on crops in Punjab, Pakistan. I. Soil physical properties and crop yield. Soil Till. Res., 59:57-65, 2001a.
ISHAQ, M.; HASSAN, A.; SAEED, M.; IBRAHIM, M. \& LAL, R. Subsoil compaction effects on crops in Punjab, Pakistan. II. Root growth and nutrient uptake of wheat and sorghum. Soil Till. Res., 60:153-161, 2001b.

JAKOBSEN, B.F. \& GREACEN, E.L. Compaction of sandy forest soils by Forwarder operations. Soil Till. Res., 5:55$70,1985$.

JORAJURIA, D. \& DRAGHI, L. Sobrecompactación del suelo agricola. Parte I: Influencia diferencial del peso y del número de pasadas. R. Bras. Eng. Agric. Amb., 4:445452,2000 .

JORAJURIA, D.; DRAGHI, L. \& ARAGON, A. The effect of vehicle weight on the distribution of compaction with depth and the yield of Lolium/Trifolium grassland. Soil Till. Res., 41:1-12, 1997.

KEMPER, W.D. \& ROSENAU, R.C. Aggregate stability and size distribution. In: KLUTE, A., ed. Methods of soil analysis. Part 1. Physical and mineralogical methods. 2.ed. Madison, Soil Science Society of America, 1986. p.425442. (SSSA Book Series)

KOGER, J.L.; BURT, E.C. \& TROUSE, A.C. Multiple pass effects of skidder tires on soil compaction. Trans. Am. Soc. Agric. Eng., 28:11-16, 1985.

LAL, R. Axle load and tillage effects on crop yields on a Mollic Ochraqualf in Northwest Ohio. Soil Till. Res., 37:143160, 1996.

LEVY, G.J. \& MILLER, W.P. Aggregate stabilities of some Southeastern U.S. soils. Soil Sci. Soc. Am. J., 61:11761182, 1997.

MARSILI, A. \& SERVADIO, P. Compaction effects of rubber or metal-tracked tractor passes on agricultural soils. Soil Till. Res., 37:37-45, 1996.

MARSILI, A.; SERVADIO, P.; PAGLIAI, M. \& VIGNOZZI, N. Changes of some physical properties of a clay soil following passage of rubber - and metal-tracked tractors. Soil Till. Res., 49:185-199, 1998.

MARTINO, D.L. \& SHAYKEWICH, C.F. Root penetration profiles of wheat and barley as affected by soil penetration resistance in field conditions. Can. J. Soil Sci., 74:193200, 1994.

NUGENT, C.; KANALI, C.; OWENDE, P.M.O.; NIEUWENHUIS, M. \& WARD, S. Characteristic site disturbance due to harvesting and extraction machinery traffic on sensitive forest sites with peat soils. For. Ecol. Manag., 180:85-98, 2003.

OLSZEVSKI, N.; BRAGA, A.P.; COSTA, L.M. \& SILVA, H.R.F. Proposição de metodologia para avaliação de degradação de pastagens em propriedades rurais. In: REUNIÃO BRASILEIRA DE MANEJO E CONSERVAÇÃO DO SOLO E DA ÁGUA,17., Fortaleza, 1998. Resumos Expandidos. Fortaleza, SBCS, 1998. p.304-305.

OUSSIBLE, M.; CROOKSTON, P.K. \& LARSON, W.E. Subsurface compaction reduces the root and shoot growth and grain yield of wheat. Agron. J., 84:34-38, 1992.

RAPER, R. \& ERBACH, D. Prediction of soil stresses using the finite element method. Trans. Am. Soc. Agric. Eng., 33:725-730, 1990. 
SEIXAS, F. \& SOUZA, C.R.S. The use of bulk density and cone penetrometer resistance as indicators to evaluate the influence of forestry machine traffic on soil compaction. In: IUFRO CONFERENCE ON INDICATORS FOR SUSTAINABLE FOREST MANAGEMENT, Melbourne, 1998. Proceedings. Melbourne, Natural Resouces and Environment, 1998. p.156-157.

SILVA, M.L.N.; CURI, N. \& BLANCANEAUX, P. Sistemas de manejo e qualidade estrutural de Latossolo Roxo. Pesq. Agropec. Bras., 35:2485-2492, 2000.

STOLF, R.; FERNANDES, J. \& FURLANI NETO, V.L. Recomendações para o uso do penetrômetro de impacto modelo IAA/PLANALSUCAR - STOLF. Araras, 1983. 9p. (Série Penetrômetro de Impacto/Boletim Técnico, 1)
TAYLOR, J.; BURT, E. \& BAILEY, N. Multipass behavior of a pneumatic tyre in tilled soils. St. Joseph, American Society of Agricultural Engineers, 1982. (ASAE Paper, 79-1549)

TISDALL, J.M. \& OADES, J.M. Organic matter and waterstable aggregates in soils. J. Soil Sci., 33:141-163, 1982.

UNGER, P.W. Organic matter and water-stable aggregate distribution in ridge-tilled surface soil. Soil Sci. Soc. Am. J., 59:1141-1145, 1995.

YEOMANS, J.C. \& BREMNER, J.M. A rapid and precise method for routine determination of organic carbon in soil. Comm. Soil Sci. Plant Anal., 19:1467-1476, 1988. 